\title{
INSTABILITY CHARACTERISTICS OF FREE-STANDING NANOWIRES BASED ON THE STRAIN GRADIENT THEORY WITH THE CONSIDERATION OF CASIMIR ATTRACTION AND SURFACE EFFECTS
}

\author{
Hamid M. Sedighi'), Hassen M. Ouakad ${ }^{2}$, Moosa Khooran') \\ 1) Shahid Chamran University of Ahvaz, Faculty of Engineering, Mechanical Engineering Department, Ahvaz 61357-43337, Iran \\ (ه.msedighi@scu.ac.ir, +986133330010 5768,moosakhooran@gmail.com) \\ 2) King Fahd University of Petroleum and Minerals, Department of Mechanical Engineering, Dhahran, 31261, KSA \\ (houakad@kfupm.edu.sa)
}

\begin{abstract}
Size-dependent dynamic instability of cylindrical nanowires incorporating the effects of Casimir attraction and surface energy is presented in this research work. To develop the attractive intermolecular force between the nanowire and its substrate, the proximity force approximation (PFA) for small separations, and the Dirichlet asymptotic approximation for large separations with a cylinder-plate geometry are employed. A nonlinear governing equation of motion for free-standing nanowires - based on the Gurtin-Murdoch model - and a strain gradient elasticity theory are derived. To overcome the complexity of the nonlinear problem in hand, a Garlerkinbased projection procedure for construction of a reduced-order model is implemented as a way of discretization of the governing differential equation. The effects of length-scale parameter, surface energy and vacuum fluctuations on the dynamic instability threshold and adhesion of nanowires are examined. It is demonstrated that in the absence of any actuation, a nanowire might behave unstably, due to the Casimir induction force.
\end{abstract}

Keywords: free-standing cylindrical nanowire, Casimir force, proximity force approximation, Dirichlet asymptotic approximation.

C 2017 Polish Academy of Sciences. All rights reserved

\section{Introduction}

In the last decades, the application of nanowire-based nanostructures is extensively growing because of their excellent physical properties such as their small size giving a large surface area-to-volume ratio. Due to their exciting properties, nanowires are promising candidates for detecting nano-objects with high sensitivity, and also they can be potentially employed in several fields, such as measurement systems [1,2], Micro/Nanoelectromechanical Systems (M/NEMS) [3], biological or gas sensing devices [4], flexible electronics and renewable energy technologies [5], integrated Shape Memory Alloy (SMA) [6], resonators and actuators [7, 8] and multifunctional NEMSs.

Essentially, when the electronic and mechanical systems were successfully integrated in a nanoscale, some new phenomena originally governed by the nanoscale quantum effects have become more and more important. As an example, the motion of a nanowire-based structure is affected by the small-scale quantum electro-dynamical interactions, such as vacuum fluctuations. The van der Waals effect stems from the electrostatic interaction between a pair of magnetic poles at an atomic-order distance. The Casimir force stands for the attractive force between two flat parallel plates of solids that originates from quantum fluctuations in the ground state of the electromagnetic field [9].

The effect of vacuum fluctuation forces can be modelled by the Casimir attraction which is the dominant phenomenon in any sub-micron separation-based application [10-12]. Using experiments, Zou et al. [13] could demonstrate the Casimir effect between two micro-machined 
silicon components on the same substrate. They achieved this result by integrating a forcesensing micromechanical beam and an electrostatic actuator on a single chip. Buks and Roukes [14] reported an experimental study of surface-surface interactions using micro-machined cantilevers fabricated from $\mathrm{Au}$ and focused on the extreme manifestation of Casimir interaction and the energy associated with this process.

The same effect is also numerically examined by Lombardo et al. [15], where the Casimir interaction energy is evaluated for configurations involving two perfectly conducting eccentric cylinders and a cylinder in front of a plane. The exact equation prescribing the Casimir force is analytically derived by Emig et al. [16] for a configuration involving a plate and a cylinder with the assumption of an intermediate geometry between the parallel plates and the plate-sphere configurations. Bordag et al. [17] published a review of some experimental and theoretical developments in the characterization of the Casimir effect in a nanoscale. They demonstrated that the Casimir force strongly depends on the shape, size, geometry and topology of boundaries. Therefore, many authors worked on computing the Casimir attraction assuming different geometries, e.g. parallel plates [18-20], plate-sphere [21], parallel cylinders [22] and plate-cylinder [23].

Also, many papers are concerned on studying the instability characteristics and nonlinear analysis of micro/nano-scale structures involving different assumptions and theories, such as the nonlocal elasticity theory [24-31], strain gradient theory [32-35], modified strain gradient theory [36, 37], strain-inertia gradient elasticity [38], nonlocal strain gradient theory [39] etc. It is worth mentioning that any nanoscale device might adhere to its actuating substrate under the effect of the Casimir attractive force, if a minimum gap between the flexible nanostructure and its substrate is not considered. Besides being one of the most effective ways of turning in the instability of freestanding nanostructures, the Casimir force can also induce undesired adhesion during the fabrication stages. Therefore, a fundamental understanding of the stability behaviour of free-standing nanowires is central to precise designing and mounting of these structures. All the previous works have studied the instability analysis of small-sized structures having planar or rectangular cross-sections. However, to the best of the authors' knowledge, no prior study considers the Casimir force while incorporating the surface effects with the application to dynamic instability characteristics of size-dependent nano-beams with a circular cross-section.

The experiments demonstrate that the surface layer plays an important role in the structural behaviour of nanoscale structures which is due to their surface-to-volume ratio [40]. Atoms in a free surface of nanostructures experience a local environment different from that met by atoms in any other bulk material. Consequently, these atoms have a different energy level than the atoms in the bulk pattern. The excess energy of surface atoms is called the surface free energy [41]. In the classical continuum mechanics, the effect of surface layer is typically ignored. However, for nanoscale devices, because of high surface-to-volume ratios, the influence of the surface layer on the overall dynamic behaviour of nanostructure could no longer be neglected. Gurtin and Murdoch [42] presented a surface elasticity theory to model the surface layer of a solid as a membrane with negligible thickness. In another study by Sedighi [43] the dynamic pull-in behaviour of an electrically actuated nano-bridge is studied, which features a rectangular cross-section incorporating the surface and intermolecular effects. Using a nonlocal finite element model, Eltaher et al. [44] examined the vibration characteristics of nano-beams accounting for the surface layer effects. Fu and Zhang [45] examined the pull-in behaviour of an electrically actuated nano-beam by incorporating the surface elasticity. They solved the complex mathematical problem by the Analog Equation Method (AEM). Their discussion considered the effects of surface energies on the static and dynamic responses, pull-in voltage and pull-in time. Koochi et al. [46] studied the influence of surface effect on the static instability behaviour of a cantilever nano-actuator in the presence of van der Waals force (vdW). 
In the previous works, Sedighi and Bozorgmehri [47, 48] studied the dynamic behaviour of vibrating nanowires based on the non-local and modified couple stress theories. In the current paper, we examine the static and dynamic instability threshold of circular cross-section-based nano-systems (such as nanowires and nanotubes) by employing the Strain Gradient Elasticity Theory (SGET) which has three length-scale parameters to account for the size dependency of sub-micron structures. In addition, we use a new Reduced-Order Method (ROM) to capture the first three natural frequencies of nanowires. The main purpose of the present study is to examine the influence of Casimir attraction on the instability/adhesion characteristics of nanobridges while addressing the size-dependency and surface layer effects. To serve this goal, the strain gradient elasticity theory together with the Euler-Bernoulli beam model are employed to derive a governing equation of nanowires accounting for the vacuum fluctuations and surface energy. To solve the nonlinear governing equation of motion, a reduced-order model, based on the so-called Galerkin expansion, is presented and performed. Finally, the effects of different parameters on the instability behaviour and adhesion time of Casimir-induced free-standing nanowires are studied.

\section{Mathematical modelling}

Figure 1 shows a schematic view and an SEM image of a free-standing doubly-clamped nanowire above a flat plate substrate. Influenced by the Casimir attractive force, the flexible cylinder can deform towards the fixed ground plane. The nanowire features an initial gap $D$, length $L$ and radius $R$. Following, the governing equations of Casimir-induced nanowires are derived based on two different approaches: the PFA approach for small separation and the Dirichlet mode approach for large separation approximations.

a)

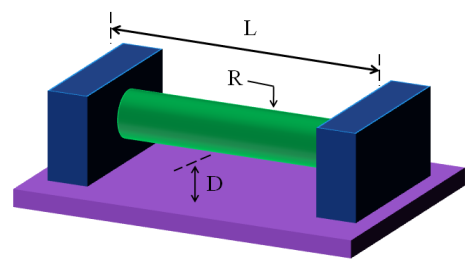

b)

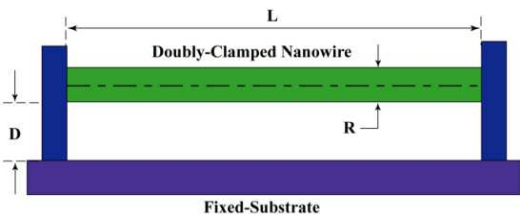

c)

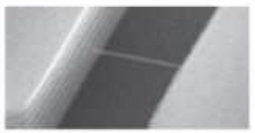

Fig. 1. A 3D schematic view (a); a 2D side view (b) and an SEM image of a doubly-clamped cylinder-plate based nanowire (c).

\subsection{Effect of surface stresses}

Figure 2 illustrates a doubly-clamped nanowire including a surface layer. Following the Gurtin-Murdoch approach [42], it is assumed that the nanowire has an elastic surface with zero thickness and specific material characteristics accounting for the surface energy effects.

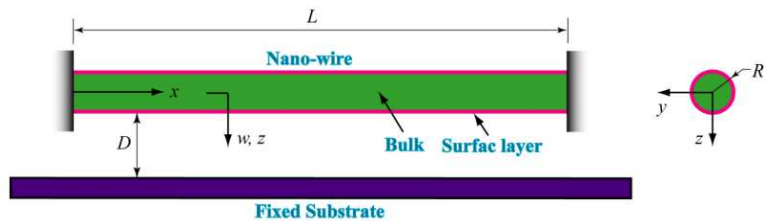

Fig. 2. A 2 D schematic representation of a doubly-clamped nanowire. 
To model the surface energy, the nano-bridge is split into two layers; a surface layer and a core (bulk) layer. The modulus of elasticity for the surface layer is represented by $E_{0}$ (determined from atomistic calculations). Assuming there is no residual stress in the bulk material due to surface tension, the corresponding bulk stress-strain relations of the beam may be written as [49]:

$$
\sigma_{x x}=E \varepsilon_{x x}+v \sigma_{z z}
$$

where $v$ is the Poisson's ratio of nano-beam. In the classical continuum mechanics theory, the stress component $\sigma_{z z}$ is assumed zero. However, the equilibrium will not be satisfied with this assumption, if the surface stress is considered. In this case, the stress component $\sigma_{z z}$ is expressed as follows [50]:

$$
\sigma_{z z}=\frac{1}{2}\left(\sigma_{z z}^{+}+\sigma_{z z}^{-}\right)+\frac{z}{2 R}\left(\sigma_{z z}^{+}-\sigma_{z z}^{-}\right),
$$

where $\sigma_{z z}^{+}$and $\sigma_{z z}^{-}$are stresses at the top and bottom surface fibres, respectively. The stresses of the surface layer satisfy the following equilibrium relation [45]:

$$
\sigma_{z z}=\frac{1}{2}\left[\left(\tau_{0}^{+}-\tau_{0}^{-}\right)\left(\frac{\partial^{2} w}{\partial x^{2}}\right)-\left(\rho_{0}^{+}-\rho_{0}^{-}\right)\left(\frac{\partial^{2} w}{\partial t^{2}}\right)\right]+\frac{z}{2 R}\left[\left(\tau_{0}^{+}+\tau_{0}^{-}\right)\left(\frac{\partial^{2} w}{\partial x^{2}}\right)-\left(\rho_{0}^{+}+\rho_{0}^{-}\right)\left(\frac{\partial^{2} w}{\partial t^{2}}\right)\right] .
$$

Moreover, Gurtin and Murdoch [42] developed the constitutive equations for the surface layers:

$$
\tau_{x x}=\tau_{0}+E_{0} \frac{\partial u}{\partial x}, \tau_{z x}=\tau_{0} \frac{\partial w}{\partial x},
$$

where $\tau_{x x}$ and $\tau_{z x}$ are non-zero membrane stresses existing on the contact surfaces of the bulk material and surface layers. The following equilibrium relations must be satisfied by stresses of the surface layers [50]:

$$
\begin{aligned}
& \left(\frac{\partial \tau_{\beta i}}{\partial \beta}\right)^{+}=\sigma_{i z}^{+}+\rho_{0}^{+}\left(\frac{\partial^{2} u_{i}}{\partial t^{2}}\right)^{+}, \\
& \left(\frac{\partial \tau_{\beta i}}{\partial \beta}\right)^{-}=\sigma_{i z}^{-}+\rho_{0}^{-}\left(\frac{\partial^{2} u_{i}}{\partial t^{2}}\right)^{-},
\end{aligned}
$$

where $\beta=x, y$ and $i=x, y, z, u_{i}^{+}=u_{i}(z=+R)$ and $u_{i}^{-}=u_{i}(z=-R)$ denote a displacement of surface layers in the $i$-direction.

\subsection{Effect of Casimir attractive force}

For the case of conducting by flat parallel plates separated with a gap $D$, the Casimir energy per unit area is expressed as [51]:

$$
E_{p p}(D)=-\frac{\pi^{2} \bar{h} c}{720 D^{3}},
$$

where $c$ is the light speed and $\bar{h}$ is Planck's constant [17]. It should be noted that this formula can be obtained while considering the electromagnetic mode structure between two plates in comparison with the free space by assigning a zero-point energy to each electromagnetic mode [52]. To predict the Casimir force in the case of a small gap, the Proximity Force Approximation (PFA) makes use of (6). The interaction between any other surfaces may be modelled by an extension of the PFA approach with the summation of infinitesimal parallel plates [17]. For small gaps, the correct zero $^{\text {th }}$-order approximation for the Casimir energy is given by [13]: 


$$
E^{P F A}=\int_{s} E_{P P}(D) d S=-\frac{\pi^{2} \bar{h} C}{720} \iint \frac{d S}{D^{3}},
$$

where $S$ is one of the two surfaces distanced by a gap. It should be emphasized that the PFA cannot be used in the case of large separation or non-smooth surfaces. Therefore, another approach should be then employed. A path integral representation [53] is used to properly model the Casimir energy for large separations. The electrodynamic Casimir energy of two parallel metallic surfaces assuming the Dirichlet mode definition at zero temperature can be expressed as $[54,55]$ :

$$
E^{D}=\frac{\bar{h} c}{2 \pi} \int_{0}^{\infty} \operatorname{Tr} \ln \left(M M_{\infty}^{-1}\right) d q_{0}
$$

where:

$$
\begin{gathered}
M_{12}\left(u, u^{\prime} ; q_{0}\right)=G_{0}\left(s_{1}(u)-s_{2}\left(u^{\prime}\right) ; q_{0}\right), \\
G_{0}\left(x, x^{\prime} ; q_{0}\right)=\frac{e^{-q_{0}\left|x-x^{\prime}\right|}}{4 \pi\left|x-x^{\prime}\right|},
\end{gathered}
$$

where a matrix $M_{12}$ denotes the geometry of surfaces 1 and 2, $M_{\infty}^{-1}$ is an inverse of matrix $M$ at infinite surface separation, $s_{i}(u)$ represents a vector referring to the $i$-th surface parameterized by a surface vector $u$ and $G_{0}$ is the free space Green function [56]. [13]:

Based on the PFA approach (for small separations), the Casimir energy can be obtained as

$$
E^{P F A}=-\frac{\pi^{3} \bar{h} c L}{960} \sqrt{\frac{R}{2 D^{5}}},
$$

where $R$ denotes a radius of nanowire and $D$ is a gap width. Therefore, the Casimir force for Small Separation Approximation (SSA) can be obtained by differentiating the energy in respect of $D$ as:

$$
f_{\text {cas }}=-\frac{\partial E(D)}{\partial D}=\frac{1}{768} \pi^{3} \bar{h} c L \sqrt{\frac{2 R}{D^{7}}}
$$

Otherwise, for the case of cylinder-plate geometry with a large separation gap, i.e. $D>>R$, the approximate expression for the attractive Casimir energy is written as [57]:

$$
E^{D}=-\frac{\bar{h} c L}{D^{2}} \frac{1}{16 \pi \ln \left(\frac{D}{R}\right)} .
$$

Therefore, the Casimir force for large separation approximation (LSA) can be expressed as:

$$
f_{c a s}=\frac{\bar{h} c L}{D^{3}} \frac{1}{8 \pi \ln \left(\frac{D}{R}\right)}+\frac{\bar{h} c L}{D^{3}} \frac{1}{16 \pi \ln ^{2}\left(\frac{D}{R}\right)} .
$$

\subsection{Strain Gradient Elasticity Theory (SGET)}

It is well-known that size has a strong impact on the mechanical behaviour of micro- and nanostructures. For instance, the bending rigidity of silica and polythene beams can increase substantially when the breadth of the beam reduces to several tens of nanometres. It should be noted that the classical continuum theories fail to predict and interpret the size effect phenomena. To address this issue, the size-dependent continuum theories (such as SGET) have been developed, which introduce additional material length-scale parameters as well as the Lame constants. In the strain gradient theory, the strain energy density with small deformation may be expressed as [58]: 
where:

$$
\bar{U}=\frac{1}{2}\left(\sigma_{i j} \varepsilon_{i j}+p_{i} \gamma_{i}+\tau_{i j k}^{(l)} \eta_{i j k}^{(1)}+m_{i j}^{s} \chi_{i j}^{s}\right)
$$

$$
\begin{gathered}
\varepsilon_{i j}=\frac{1}{2}\left(u_{i, j}+u_{j, i}\right), \\
\gamma_{i}=\varepsilon_{m m, i}, \\
\eta_{i j k}^{(I)}=\frac{1}{3}\left(\varepsilon_{j k, i}+\varepsilon_{k i, j}+\varepsilon_{i j, k}\right)-\frac{1}{15} \delta_{i j}\left(\varepsilon_{m m, k}+2 \varepsilon_{m k, m}\right)-\frac{1}{15}\left[\delta_{j k}\left(\varepsilon_{m m, i}+2 \varepsilon_{m i, m}\right)+\delta_{k i}\left(\varepsilon_{m m, j}+2 \varepsilon_{m j, m}\right)\right], \\
\chi_{i j}^{s}=\frac{1}{2} e_{j k l} u_{l, k i} .
\end{gathered}
$$

In the above equations, $u_{i}, \gamma_{i}, \eta_{i j k}^{(1)}, \chi_{i j}^{s}, \delta_{i j}$ and $e_{i j k}$ indicate a displacement vector, a dilatation gradient vector, a deviatory stretch gradient tensor, a symmetric rotation gradient tensor, Kronecker delta and permutation symbols, respectively. Also, $\varepsilon_{i j}, \sigma_{i j}, p_{i}, \tau_{i j k}^{(1)}, m_{i j}^{s}$, are a strain tensor, Cauchy's stress and high order stress tensors, respectively, and are expressed as [58]:

$$
\begin{gathered}
\sigma_{i j}=2 \mu\left(\varepsilon_{i j}+\frac{v}{1-2 v} \varepsilon_{m m} \delta_{i j}\right), \\
p_{i}=2 \mu l_{0}^{2} \gamma_{i}, \\
\tau_{i j k}^{(1)}=2 \mu l_{1}^{2} \eta_{i j k}^{(1)}, \\
m_{i j}^{s}=2 \mu l_{2}^{2} \chi_{i j}^{s},
\end{gathered}
$$

where $v$ and $\mu$ are Poisson's ratio and shear modulus, respectively. Also, $l_{0}, l_{1}$ and $l_{2}$ are additional material length-scale parameters in the constitutive equations of higher-order stresses.

\subsection{Governing equation of motion}

In this section, the governing equation for size-dependent vibration of Casimir-induced nanowires in the presence of surface effects is derived. Based on the Euler-Bernoulli beam theory, the displacement field can be expressed as follows:

$$
u(x, z, t)=-z \theta(x, t)=-z \frac{\partial w}{\partial x}, \quad w=w(x, t) .
$$

The strain of a material point located at a distance $z$ from the top plane for a nano-beam is represented as:

$$
\varepsilon_{x x}=\frac{\partial u}{\partial x}=-z \frac{\partial^{2} w}{\partial x^{2}} .
$$

Substituting the linear displacement field of (23) into (15) to (22), the total strain energy on the basis of SGET for a deformed Euler-Bernoulli beam is given by:

$$
U_{S G E T}=\frac{1}{2} \int_{0}^{L}\left[\left(2 \mu A l_{0}^{2}+\frac{8}{15} \mu A l_{1}^{2}+\mu A l_{2}^{2}\right)\left(\frac{\partial^{2} w}{\partial x^{2}}\right)^{2}+I\left(2 \mu l_{0}^{2}+\frac{4}{5} \mu l_{1}^{2}\right)\left(\frac{\partial^{3} w}{\partial x^{3}}\right)^{2}\right] d x .
$$

In (25), $I$ represents the second moment of inertia of the beam around its $y$-axis. Based on the surface elasticity theory, the strain energy in the surface layer with zero thickness can be expressed as [59]: 


$$
U_{s}=\frac{1}{2} \int_{0}^{L} \int_{S}\left(\tau_{\alpha \beta} \varepsilon_{\alpha \beta}+\tau_{k \alpha} u_{k, \alpha}\right) d S d x
$$

where $\alpha, \beta$ denote in-plane Cartesian coordinates of the surface and $k$ is an out-plane Cartesian coordinate of the surface. Substituting (4) into (26) results in:

$$
\begin{aligned}
U_{s}= & \frac{1}{2} \int_{0}^{L} \int_{S}\left[\left(\tau_{0}+E_{0} \frac{\partial u}{\partial x}\right) \varepsilon_{x x}+\tau_{0}\left(\frac{\partial w}{\partial x}\right)^{2}\right] d S d x \\
= & \frac{1}{2} \int_{0}^{L} \int_{S^{+}}\left[-\left(\tau_{0}^{+}-E_{0}^{+} z \frac{\partial^{2} w}{\partial x^{2}}\right) z \frac{\partial^{2} w}{\partial x^{2}}+\tau_{0}^{+}\left(\frac{\partial w}{\partial x}\right)^{2}\right] d S^{+} d x \\
& +\frac{1}{2} \int_{0}^{L} \int_{S^{-}}\left[-\left(\tau_{0}^{-}-E_{0}^{-} z \frac{\partial^{2} w}{\partial x^{2}}\right) z \frac{\partial^{2} w}{\partial x^{2}}+\tau_{0}^{-}\left(\frac{\partial w}{\partial x}\right)^{2}\right] d S^{-} d x .
\end{aligned}
$$

Simplifying the surface energy described in (27) yields:

$$
U_{s}=\frac{1}{2} \int_{0}^{L}\left[\left(\tau_{0}^{+}+\tau_{0}^{-}\right) S_{0}^{*}\left(\frac{\partial w}{\partial x}\right)^{2}+\left(E_{0}^{+}+E_{0}^{-}\right) I_{0}^{*}\left(\frac{\partial^{2} w}{\partial x^{2}}\right)^{2}\right] d x .
$$

In addition, the virtual work $\tilde{W}$ induced by the axial and Casimir forces incorporating the vonKármán type nonlinear strain can be written as [60]:

$$
\tilde{W}=-\frac{1}{2} \int_{0}^{L}\left(N_{0}+\frac{E A}{2 L} \int_{0}^{l}\left(\frac{\partial w}{\partial x}\right)^{2} d x\right)\left(\frac{\partial w}{\partial x}\right)^{2} d x+\int_{0}^{L} f_{\text {cas }} w d x .
$$

The kinetic energies for the bulk material and surface layer are obtained as follows:

$$
\begin{aligned}
T & =\frac{1}{2} \int_{0}^{L} \int_{A} \rho\left(\frac{\partial w}{\partial t}\right)^{2} d A d x+\frac{1}{2} \int_{0}^{L} \int_{S^{+}} \rho_{0}^{+}\left(\frac{\partial w}{\partial t}\right)^{2} d S^{+} d x+\frac{1}{2} \int_{0}^{L} \int_{S^{-}} \rho_{0}^{-}\left(\frac{\partial w}{\partial t}\right)^{2} d S^{-} d x \\
& =\frac{1}{2}\left(\rho A+\left(\rho_{0}^{+}+\rho_{0}^{-}\right) S_{0}^{*}\right) \int_{0}^{L}\left(\frac{\partial w}{\partial t}\right)^{2} d x .
\end{aligned}
$$

The first variation of the strain energy function is obtained as [61, 62]:

$$
\delta U_{b}=\int_{0}^{L} \int_{A} \sigma_{i j} \delta \varepsilon_{i j} d A d x
$$

where $A$ is an area of the cross-section of nano-beam.

Substituting (15) into (22), the variation of the strain energy function can be obtained as:

$$
\delta U_{b}=-\int_{0}^{l} \int_{A} z \sigma_{x x} \frac{\partial^{2} \delta w}{\partial x^{2}} d A d x=\int_{0}^{l} M(x, t)\left(\frac{\partial^{2} \delta w}{\partial x^{2}}\right) d x,
$$

where $M(x, t)=-\int_{A} \sigma_{x x} z d A$ is a resultant bending moment of the nanowire. Applying the Hamilton's principle results in:

$$
\int_{t_{1}}^{t_{2}}\left(\delta T-\left[\delta U_{b}+\delta U_{s}\right]+\delta W\right) d t=0 .
$$

Subsequently, while assuming some mathematical simplifications, the nonlinear governing equation of motion can be written as follows: 


$$
\begin{aligned}
\frac{\partial^{2} M}{\partial x^{2}}= & -\left(\rho A+\left(\rho_{0}^{+}+\rho_{0}^{-}\right) S_{0}^{*}\right) \frac{\partial^{2} w}{\partial t^{2}}-\left(E_{0}^{+}+E_{0}^{-}\right) I_{0}^{*} \frac{\partial^{4} w}{\partial x^{4}}+\left(\tau_{0}^{+}+\tau_{0}^{-}\right) S_{0}^{*} \frac{\partial^{2} w}{\partial x^{2}} \\
& +\left(2 \mu A l_{0}^{2}+\frac{8}{15} \mu A l_{1}^{2}+\mu A l_{2}^{2}\right) \frac{\partial^{4} w}{\partial x^{4}}-I\left(2 \mu l_{0}^{2}+\frac{4}{5} \mu l_{1}^{2}\right) \frac{\partial^{6} w}{\partial x^{6}} \\
& +\left(N_{0}+\frac{E A}{2 L} \int_{0}^{L}\left(\frac{\partial w}{\partial x}\right)^{2} d x\right) \frac{\partial^{2} w}{\partial x^{2}}+f_{c a s},
\end{aligned}
$$

and the corresponding boundary conditions at the ends of the nano-bridge can be written as follows:

$$
w(x=0)=0, \frac{\partial w}{\partial x}(x=0)=0, w(x=L)=0, \frac{\partial w}{\partial x}(x=L)=0,
$$

Using the relation of normal stress described in the equation (1), a resultant bending moment of nanowire can be expressed as:

$$
\begin{aligned}
M(x, t)= & -\int_{A} \sigma_{x x} z d A=-\int_{A}\left(E z \varepsilon_{x x}+v\left\{\frac{z}{2}\left[\left(\tau_{0}^{+}-\tau_{0}^{-}\right)\left(\frac{\partial^{2} w}{\partial x^{2}}\right)-\left(\rho_{0}^{+}-\rho_{0}^{-}\right)\left(\frac{\partial^{2} w}{\partial t^{2}}\right)\right]\right.\right. \\
& \left.\left\{+\frac{z^{2}}{h}\left[\left(\tau_{0}^{+}+\tau_{0}^{-}\right)\left(\frac{\partial^{2} w}{\partial x^{2}}\right)-\left(\rho_{0}^{+}+\rho_{0}^{-}\right)\left(\frac{\partial^{2} w}{\partial t^{2}}\right)\right]\right\}\right) d A \\
= & E I \frac{\partial^{2} w}{\partial x^{2}}-\frac{v A R}{2}\left(\left(\tau_{0}^{+}-\tau_{0}^{-}\right) \frac{\partial^{2} w}{\partial x^{2}}-\left(\rho_{0}^{+}-\rho_{0}^{-}\right) \frac{\partial^{2} w}{\partial t^{2}}\right)-\frac{v I}{2 R}\left(\left(\tau_{0}^{+}+\tau_{0}^{-}\right) \frac{\partial^{2} w}{\partial x^{2}}-\left(\rho_{0}^{+}+\rho_{0}^{-}\right) \frac{\partial^{2} w}{\partial t^{2}}\right) .
\end{aligned}
$$

Therefore, the governing equation of motion for nanowires can be obtained by inserting (27) into (25) as follows:

$$
\begin{aligned}
& E I \frac{\partial^{4} w}{\partial x^{4}}+\left(2 \mu A l_{0}^{2}+\frac{8}{15} \mu A l_{1}^{2}+\mu A l_{2}^{2}\right) \frac{\partial^{4} w}{\partial x^{4}}-I\left(2 \mu l_{0}^{2}+\frac{4}{5} \mu l_{1}^{2}\right) \frac{\partial^{6} w}{\partial x^{6}} \\
&-\frac{v I}{2 R}\left(\left(\tau_{0}^{+}+\tau_{0}^{-}\right) \frac{\partial^{4} w}{\partial x^{4}}-\left(\rho_{0}^{+}+\rho_{0}^{-}\right) \frac{\partial^{4} w}{\partial x^{2} \partial t^{2}}\right)-\frac{v A R}{2}\left(\left(\tau_{0}^{+}-\tau_{0}^{-}\right) \frac{\partial^{4} w}{\partial x^{4}}-\left(\rho_{0}^{+}-\rho_{0}^{-}\right) \frac{\partial^{4} w}{\partial x^{2} \partial t^{2}}\right) \\
&+\left(E_{0}^{+}+E_{0}^{-}\right) I_{0}^{*} \frac{\partial^{4} w}{\partial x^{4}}-\left(\tau_{0}^{+}+\tau_{0}^{-}\right) S_{0}^{*} \frac{\partial^{2} w}{\partial x^{2}}=-\left(\rho A+\left(\rho_{0}^{+}+\rho_{0}^{-}\right) S_{0}^{*}\right) \frac{\partial^{2} w}{\partial t^{2}} \\
&+\left.\left(N_{0}+\frac{E A}{2 L} \int\left(\frac{\partial w}{\partial x}\right)^{2} d x\right) \frac{\partial^{2} w}{\partial x^{2}}+f_{c a s}\right] .
\end{aligned}
$$

\subsection{Normalization of governing equation of motion}

In order to describe the governing equation in a non-dimensional from, the following variables are introduced:

$$
\begin{aligned}
& W=\frac{w}{D}, \xi=\frac{x}{L}, \alpha=\frac{E A D^{2}}{2 E I}, \tau=\sqrt{\frac{E^{+} h^{2}}{12 \rho^{+} L^{4}}} t, f_{i}=\frac{N_{0} L^{2}}{E I}, k=\frac{D}{R}, \mu_{l}=\frac{12 \mu}{E(h / l)^{2}} \\
& \mu=\frac{\left(E_{0}^{+}+E_{0}^{-}\right) I_{0}^{*}}{E^{+} I}, \sigma_{1}=\frac{-12 A R v\left(\tau_{0}^{+}+\tau_{0}^{-}\right)-36 v A R\left(\tau_{0}^{+}-\tau_{0}^{-}\right)}{E^{+} I} \sigma_{2}=\frac{\left(\tau_{0}^{+}+\tau_{0}^{-}\right) S_{0}^{*} l^{2}}{E^{+} I}, \\
& \kappa_{1}=\frac{2 v\left(\rho_{0}^{+}+\rho_{0}^{-}\right) R+3\left(\rho_{0}^{+}-\rho_{0}^{-}\right)}{12 \rho^{+} L^{2}}, \kappa_{2}=\frac{\left(\rho_{0}^{+}+\rho_{0}^{-}\right) S_{0}^{*}}{\rho^{+} A}, \gamma=\frac{\bar{h} c L^{4}}{8 \pi D^{4} E I} \\
& \alpha_{4}=\left(1+\frac{\mu_{l}}{15}\left(30\left(\frac{l_{0}}{l_{2}}\right)^{2}+8\left(\frac{l_{1}}{l_{2}}\right)^{2}+15\right)+\mu-\sigma_{1}\right), \alpha_{6}=\frac{\mu_{l}}{30(L / h)^{2}}\left[5\left(\frac{l_{0}}{l_{2}}\right)^{2}+2\left(\frac{l_{1}}{l_{2}}\right)^{2}\right]
\end{aligned}
$$


Thus, the non-dimensional equation of motion for size-dependent nanowires incorporating the surface effects and the Casimir force can be written as:

$$
\alpha_{4} \frac{\partial^{4} W}{\partial \xi^{4}}-\alpha_{6} \frac{\partial^{6} W}{\partial \xi^{6}}+\kappa_{1} \frac{\partial^{4} W}{\partial \xi^{2} \partial \bar{\tau}^{2}}-\sigma_{2} \frac{\partial^{2} W}{\partial \xi^{2}}=\left[\frac{\pi^{4} \gamma}{96} \sqrt{\frac{2}{k(1-W)^{7}}}+\alpha \frac{\partial^{2} W}{\partial \xi^{2}} \int_{0}^{1}\left(\frac{\partial W}{\partial \xi}\right)^{2} d \xi-\left(1+\kappa_{2}\right) \frac{\partial^{2} W}{\partial \bar{\tau}^{2}}\right],
$$

for the SSA approximation and:

$$
\begin{aligned}
& W=\frac{w}{D}, \xi=\frac{x}{L}, \alpha=\frac{E A D^{2}}{2 E I}, \tau=\sqrt{\frac{E^{+} h^{2}}{12 \rho^{+} L^{4}}} t, f_{i}=\frac{N_{0} L^{2}}{E I}, k=\frac{D}{R}, \mu_{l}=\frac{12 \mu}{E(h / l)^{2}} \\
& \mu=\frac{\left(E_{0}^{+}+E_{0}^{-}\right) I_{0}^{*}}{E^{+} I}, \sigma_{1}=\frac{-12 A R v\left(\tau_{0}^{+}+\tau_{0}^{-}\right)-36 v A R\left(\tau_{0}^{+}-\tau_{0}^{-}\right)}{E^{+} I} \sigma_{2}=\frac{\left(\tau_{0}^{+}+\tau_{0}^{-}\right) S_{0}^{*} l^{2}}{E^{+} I} \text {, } \\
& \kappa_{1}=\frac{2 v\left(\rho_{0}^{+}+\rho_{0}^{-}\right) R+3\left(\rho_{0}^{+}-\rho_{0}^{-}\right)}{12 \rho^{+} L^{2}}, \quad \kappa_{2}=\frac{\left(\rho_{0}^{+}+\rho_{0}^{-}\right) S_{0}^{*}}{\rho^{+} A}, \gamma=\frac{\bar{h} c L^{4}}{8 \pi D^{4} E I} \\
& \alpha_{4}=\left(1+\frac{\mu_{l}}{15}\left(30\left(\frac{l_{0}}{l_{2}}\right)^{2}+8\left(\frac{l_{1}}{l_{2}}\right)^{2}+15\right)+\mu-\sigma_{1}\right), \quad \alpha_{6}=\frac{\mu_{l}}{30(L / h)^{2}}\left[5\left(\frac{l_{0}}{l_{2}}\right)^{2}+2\left(\frac{l_{1}}{l_{2}}\right)^{2}\right] \\
& \alpha_{4} \frac{\partial^{4} W}{\partial \xi^{4}}-\alpha_{6} \frac{\partial^{6} W}{\partial \xi^{6}}+\kappa_{1} \frac{\partial^{4} W}{\partial \xi^{2} \partial \bar{\tau}^{2}}-\sigma_{2} \frac{\partial^{2} W}{\partial \xi^{2}}= \\
& {\left[\frac{\gamma}{(1-W)^{3}}\left(\frac{1}{\ln (k(1-W))}+\frac{1}{2(\ln (k(1-W)))^{2}}\right)+\alpha \frac{\partial^{2} W}{\partial \xi^{2}} \int_{0}^{1}\left(\frac{\partial W}{\partial \xi}\right)^{2} d \xi-\left(1+\kappa_{2}\right) \frac{\partial^{2} W}{\partial \bar{\tau}^{2}}\right]}
\end{aligned}
$$

for the LSA approach. It is worth mentioning that, due to the nanowire deflection $W(x, t)$, a distance $D$ assumed in the Casimir force is replaced by $D-W$. Moreover, in the case of a large separation gap, it is assumed that $D+R \approx D$.

\section{Solution methodology}

\subsection{Reduced-order modelling}

To simulate the total response of a nanowire, (39a) and (39b) are discretized using a socalled Galerkin procedure to yield a Reduced-Order Model (ROM). Hence, the deflection of nanowire is approximated as:

$$
W(x, t)=\sum_{i=1}^{n} u_{i}(t) \phi_{i}(x)
$$

where $\phi_{i}(x)$ are normalized linear undamped mode shapes of a perfectly straight clamped beam, and $u_{i}(t)$ are non-dimensional modal coordinates. It is worth mentioning here that, because of a nonlinear nature of the Casimir attractive force, symmetric (odd) and antisymmetric (even) mode shapes are assumed in this work. To obtain the ROM, we substitute the equation (40) into (39a) and (39b), multiply by $\phi_{i}(x)$, use the orthogonality conditions of the mode shapes, and then integrate the outcome from 0 to 1 . The results are differential equations in terms of the modal coordinates $u_{i}(t)$ that can be integrated and the total response of nanowire can be obtained from (40).

Several issues need to be clarified about the developed ROM. Unlike the case of rectangular micro-beams [63], here there is no numerical advantage of multiplying (39a) or (39b) by the denominator of Casimir force term, since the mode shape $\phi_{i}(x)$ will remain embedded in the nonlinear terms even after imposing the orthogonality of the mode shapes. To deal with the 
complicated integral terms due to the nonlinear Casimir attractive force, we evaluated the spatial integrals containing the $\phi_{i}(x)$ terms numerically, using a trapezoidal method, while simultaneously integrating the differential equations of the modal coordinates $u_{i}(t)$.

\subsection{Static response}

To simulate the static response, all time-dependent terms in the governing differential equations of nanowire in (39a) and (39b) are set equal to zero. Then, the modal coordinates $u_{i}(t)$ are replaced by unknown constant coefficients $a_{i}(i=1,2, \ldots, n)$. This results in a system of nonlinear algebraic equations in terms of coefficients $a_{i}$. The system is then solved numerically using the Newton-Raphson method [64] to obtain the static deflection of nanowire.

\subsection{Eigen-value problem}

In this work, we propose to examine the variation of fundamental natural frequency of a nanowire under the effect of the attractive force. Toward this, we consider the ROM obtained in Subsection 3.1, which can be re-written in a matrix form as:

$$
M(U) \dot{U}=R(U),
$$

where:

$$
U=\left[u_{1}, u_{2}, u_{3}, \ldots, u_{n}\right],
$$

is a modal vector of amplitudes, $M(U)$ is a nonlinear matrix representing the coefficients of $\dot{U}$, and $R(U)$ is a right-hand side vector representing the forcing, stiffness, and damping coefficients. Both $M(U)$ and $R(U)$ are nonlinear functions of the modal coordinates $u_{i}(t)$. Note here that we consider the symmetric and anti-symmetric mode shapes in the ROM to get all the possible natural frequencies and mode shapes of the nanowire.

Next, we split $U$ into a static component $X_{s}$, representing the equilibrium position due to the applied Casimir force, and a dynamic component $\eta(t)$ representing the perturbation around the equilibrium position, that is:

$$
U=X_{s}+\eta(t) \text {. }
$$

Then, substituting (43) into (41), using the Taylor series expansion assuming small $\eta$, eliminating the quadratic terms, and using the fact that $R\left(X_{s}\right)=0$, we obtain the following:

$$
M\left(X_{s}\right) \dot{\eta}=J\left(X_{s}\right) \eta
$$

where $J\left(X_{s}\right)$ is a Jacobian matrix calculated at the equilibrium points [65].

To calculate the natural frequencies of nanowire for the applied attractive force, we substitute the stable static solution, $X_{s}$, into the matrix $M^{-1} J$ and then find its corresponding eigenvalues. The eigenvalues are calculated by solving the below equation, which gives a characteristic algebraic equation for an eigenvalue $\lambda$ :

$$
\operatorname{det}\left(M^{-1}\left(X_{s}\right) J\left(X_{s}\right)-\lambda I\right)=0
$$

where $I$ symbolizes an identity matrix. Finally, by taking the square root of each individual eigenvalue, we obtain the natural frequencies of the system. 


\section{Results and discussion}

This work attempts to examine the influence of vacuum fluctuations and size-dependency on the nonlinear dynamic behaviour of freestanding nanowires. In order to endorse the accuracy of present ROM simulations, the static deflection of nanowire versus the Casimir force for SSA and LSA models are plotted and compared with the results of Farrokhabadi et al. [66] (see Fig. 3). One can clearly observe that the results of our modelling are in a good agreement with those reported in the literature.

a)

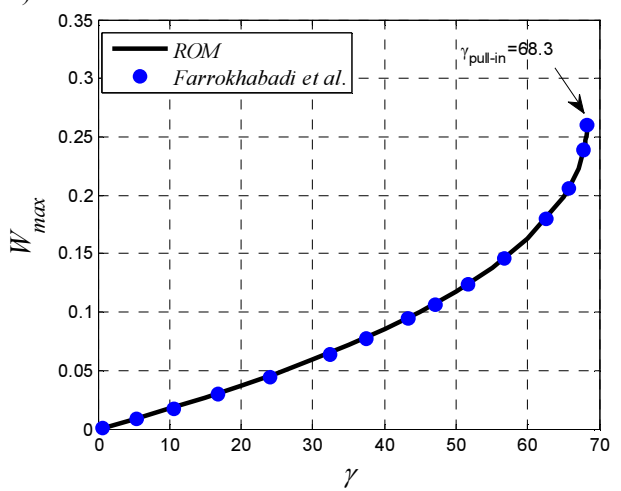

b)

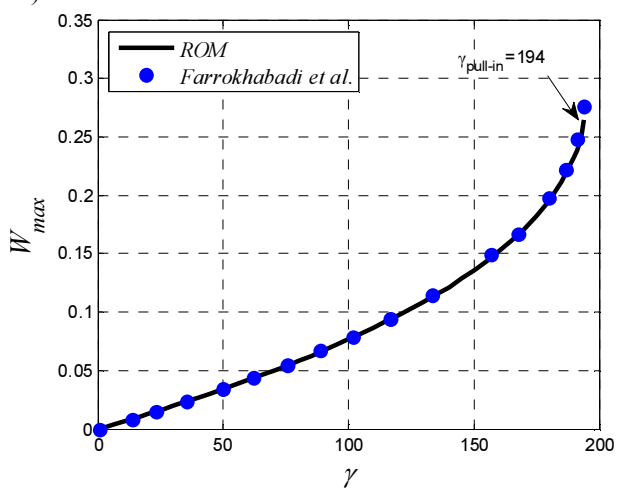

Fig. 3. Variations of the static deflection vs the Casimir force for (a) SSA approximation $(k=5)$ and (b) LSA approximation $(k=100)$, and for $\alpha_{4}=1, \alpha_{6}, \kappa_{1}, \sigma_{2}, \alpha, \kappa_{2}=0$.

To show the instability behaviour of a nano-fabricated system by varying the Casimir parameter, Fig. 4 displays variations of the static deflection and the fundamental frequency of nanowire versus the Casimir force parameter $\gamma$ for the following system parameters: $\alpha_{1}=6 ; \kappa_{1}=0 ; \kappa_{2}=0.2$. It is worth mentioning that, when the geometry parameter $k$ takes rather large values (here $k=100$ ), the LSA approximations should be employed to take the Casimir induction force into account. One can observe that any increase in the Casimir induction force leads to reduction of the initial gap and natural frequency of nanowire up to the critical point $(\gamma=216)$ beyond which the nanowire becomes unstable and consequently it collapses onto the fixed substrate. Moreover, Fig. 5 indicates the same behaviour when the geometry parameter is considered small, in which we have to assume the SSA approximation to account for the vacuum fluctuations. It is demonstrated that, for SSA approximation, the nanowire falls to the fixed plate at lower values of the Casimir parameter $\gamma$.

In order to examine the influence of geometry ratio $k$ on the predicted critical Casimir value of induced nanowires, variations of $\gamma_{\text {pull-in }}$ as functions of the geometry parameter for two different models, i.e. LSA and SSA, are displayed in Fig. 6. One can observe that $\gamma_{\text {pull-in }}$ is remarkably affected by this parameter. It is shown that the Casimir critical value increases continuously with increasing the geometry ratio $k$. This reveals that, as the initial gap $D$ increases and/or the radius of nanowire decreases, the free-standing nanowire diverges to the bottom plate at higher values of the Casimir parameter.

Due to a high surface-to-volume ratio of several nano-fabricated structures, it was empirically showed that the surface stress plays a significant role in the static and dynamic behaviour of such devices. To show the impact of surface stress $\sigma_{2}$ on the instability characteristics of Casimir-induced nanowires, variations of the static deflection and the first 
natural frequency are presented in Figs. 7 and 8 for both LSA and SSA approximations, respectively.

a)

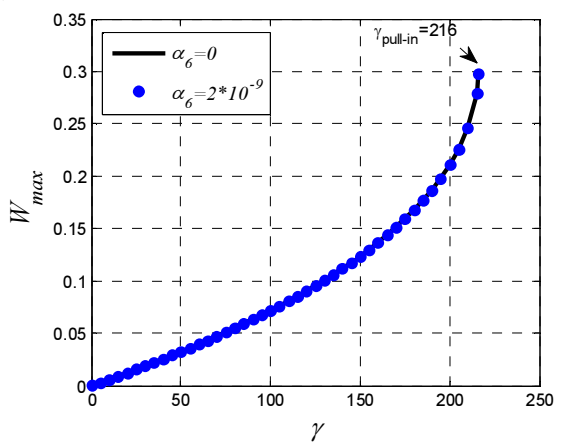

b)

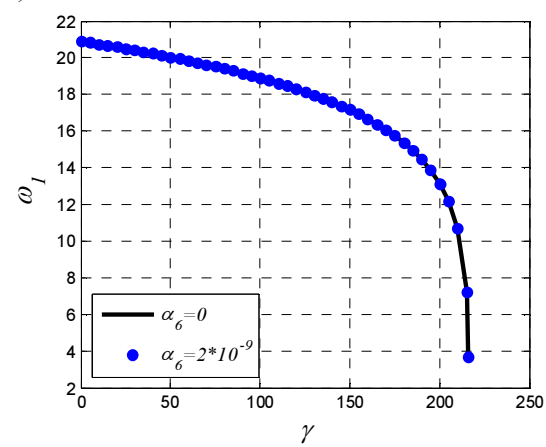

Fig. 4. Variations of (a) the static deflection and (b) the natural frequency vs the Casimir force for LSA approximation and $k=100, \alpha_{4}=1, \sigma_{2}=2$

a)

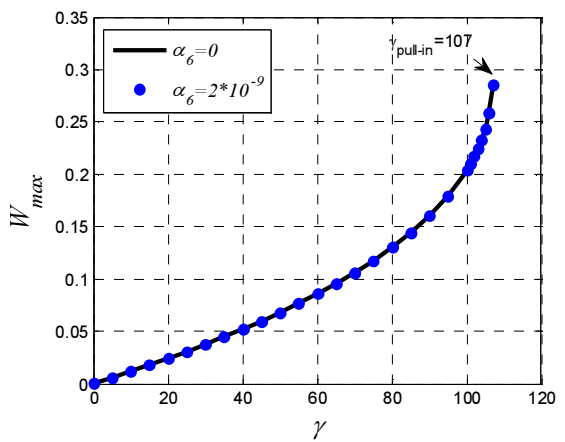

b)

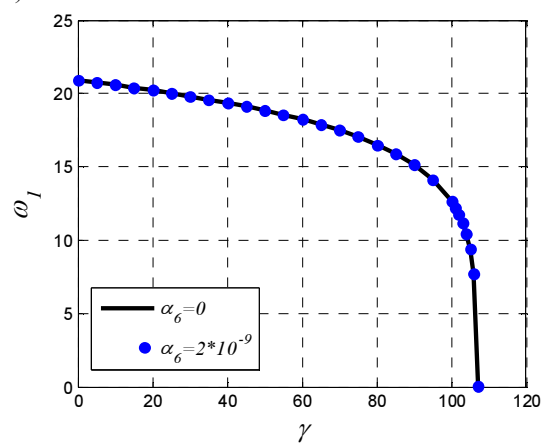

Fig. 5. Variations of (a) the static deflection and (b) the natural frequency vs the Casimir force for SSA approximation and $k=10, \alpha_{4}=1, \sigma_{2}=2$

a)

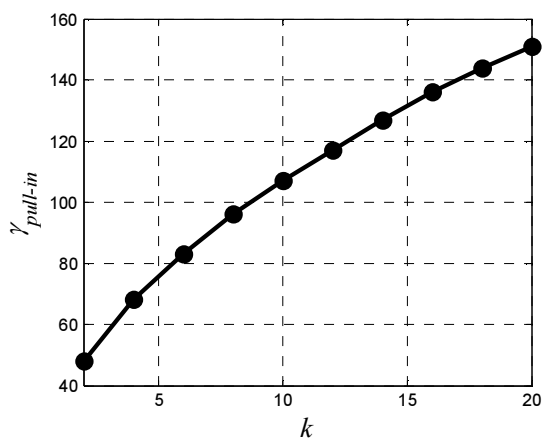

b)

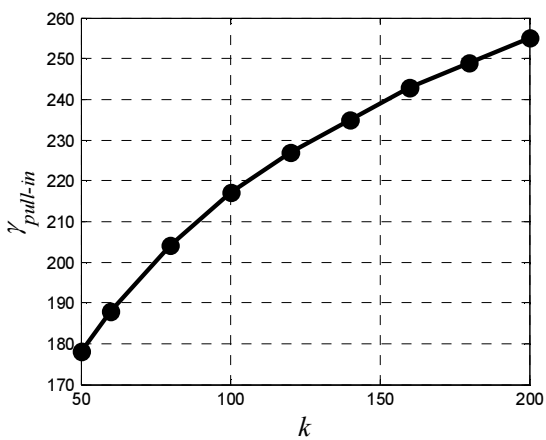

Fig. 6. Variations of the critical Casimir value vs the geometry ratio for (a) SSA and (b) LSA approximations.

It is illustrated that the critical values of Casimir parameter, predicted by both LSA and SSA models, increase as the parameter $\sigma_{2}$ varies from negative to positive values. In addition, 
one can infer that in the absence of any Casimir attraction, the fundamental frequency of nanowire shifts upward by increasing the surface stress values.

a)

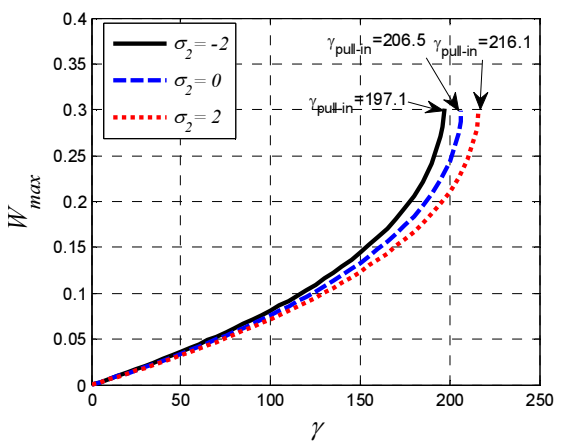

b)

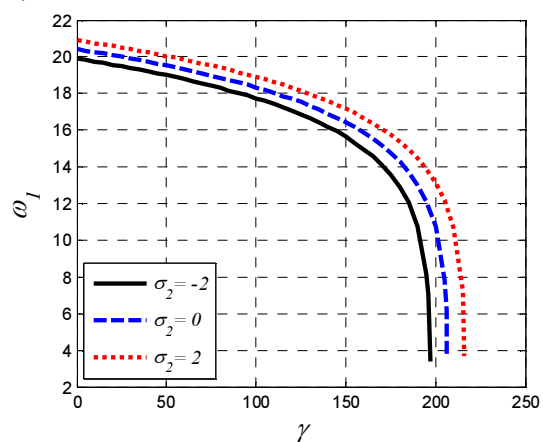

Fig. 7. Variations of (a) the static deflection and (b) the natural frequency vs the Casimir force for LSA approximation and some assigned values of $\sigma_{2}$ and $\alpha_{4}=1$.

a)

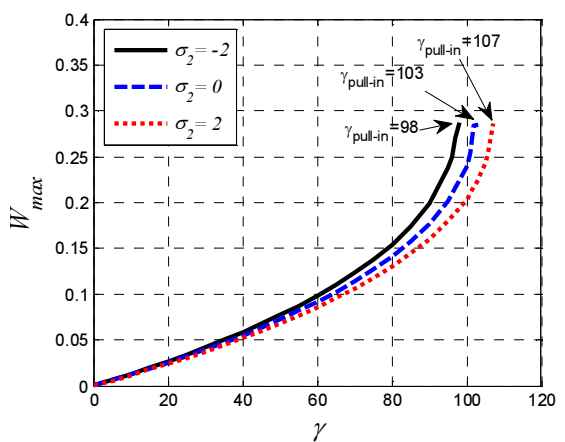

b)

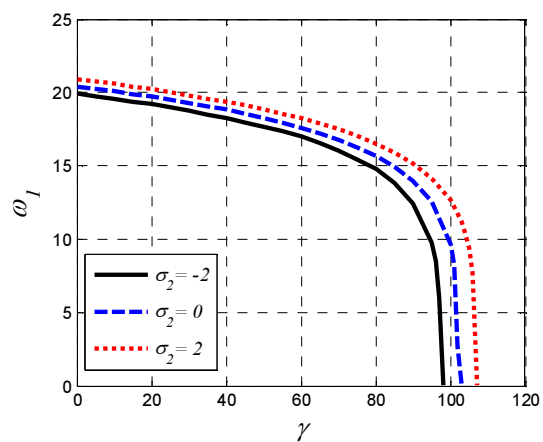

Fig. 8. Variations of (a) the static deflection and (b) the natural frequency vs the Casimir force for SSA approximation and some assigned values of $\sigma_{2}$ and $\alpha_{4}=1$.

As mentioned earlier, it has been validated that the mechanical behaviour of miniaturesized structures are size-dependent and the classical theories may not predict the instability threshold of nano-beams precisely. To this end, the strain gradient elasticity theory (SGET) including three material length-scale parameters has been employed to capture the microstructure size-dependency. In this paper, the non-dimensional parameter $\alpha_{4}$ accounts for the length-scale parameters. Figs. 9 and 10 depict variations of the initial gap between the nanowire and substrate as well as the first natural frequency of induced nanowire as functions of the Casimir parameter $\gamma$ for three values of parameter $\alpha_{4}$. One can conclude that the instability occurs at higher values of Casimir parameter by increasing the size parameter $\alpha_{4}$. Moreover, according to Figs. $9 \mathrm{~b}$ and $10 \mathrm{~b}$, it is clearly shown that the fundamental frequency of nanowire increases when the length-scale parameter increases. 
a)

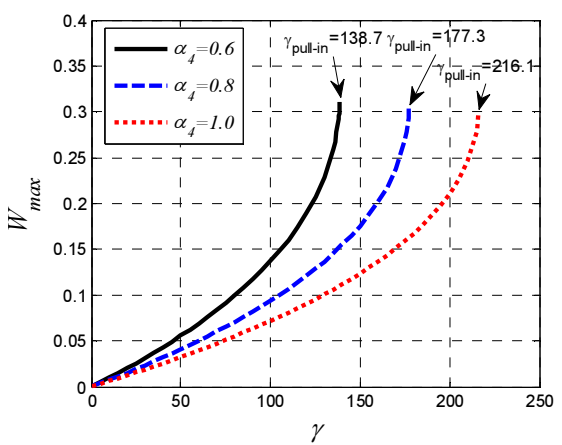

b)

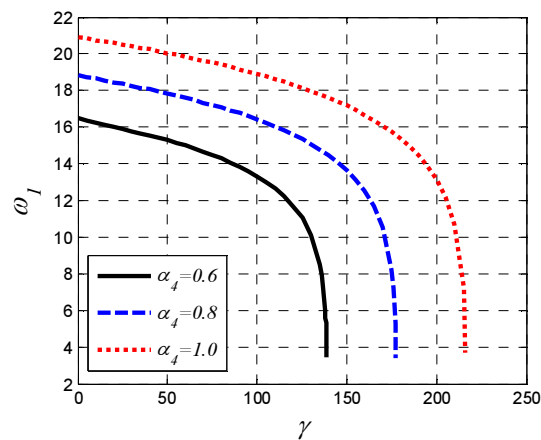

Fig. 9. Variations of (a) the static deflection and (b) the natural frequency vs the Casimir force for LSA approximation and $\sigma_{2}=2$

a)

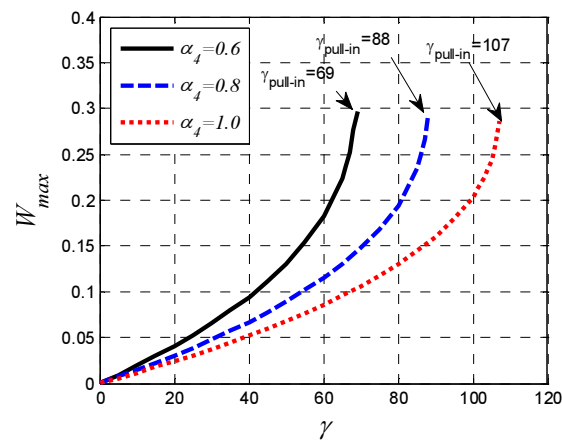

b)

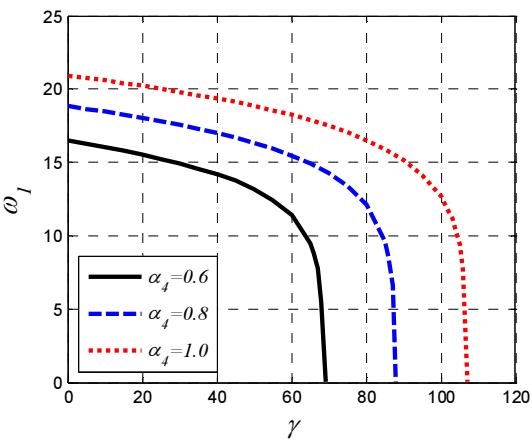

Fig. 10. Variations of (a) the static deflection and (b) the natural frequency vs the Casimir force for SSA approximation and $\sigma_{2}=2$

a)

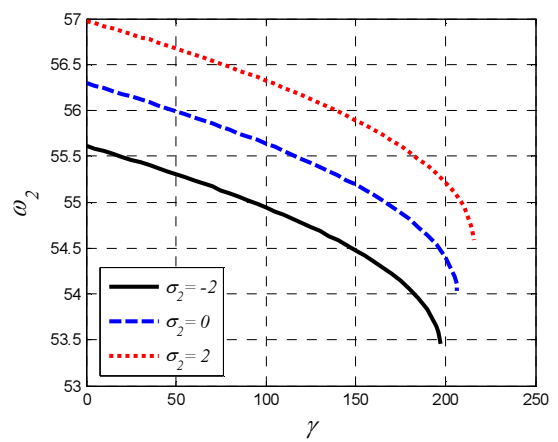

b)

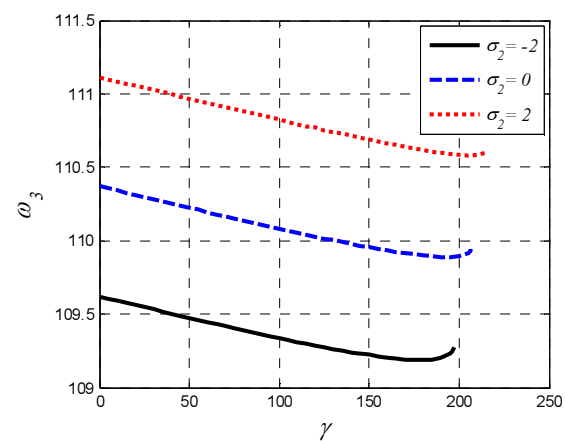

Fig. 11. Variations of (a) the second and (b) the third natural frequencies vs the Casimir force for LSA approximation and some assigned values of $\sigma_{2}$ and $\alpha_{4}=1$. 
a)

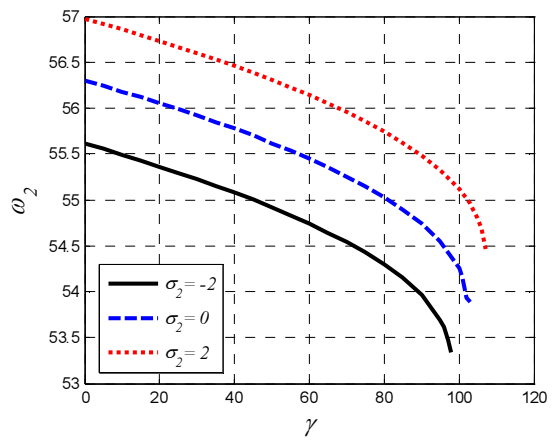

b)

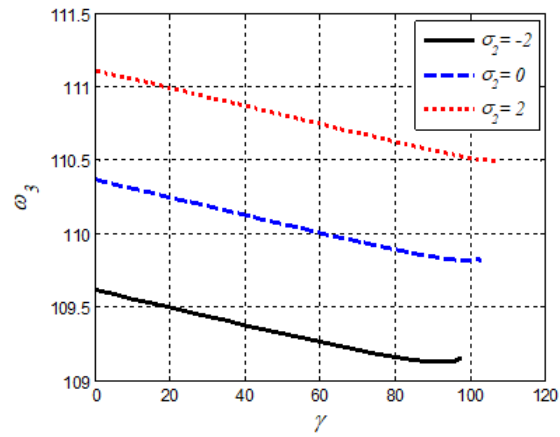

Fig. 12. Variations of (a) the second and (b) the third natural frequencies vs the Casimir force for SSA approximation and some assigned values of $\sigma_{2}$ and $\alpha_{4}=1$

a)

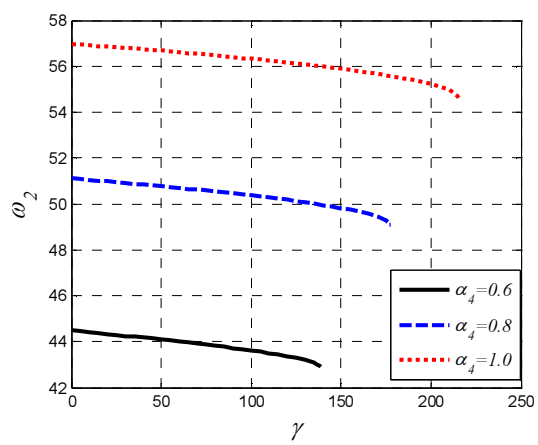

b)

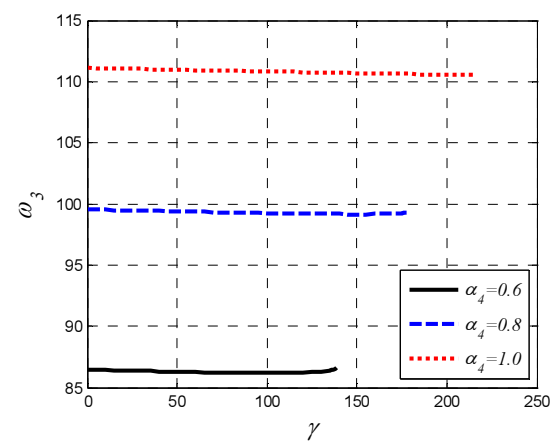

Fig. 13. Variations of (a) the second and (b) the third natural frequencies vs the Casimir force for LSA approximation and $\sigma_{2}=2$

a)

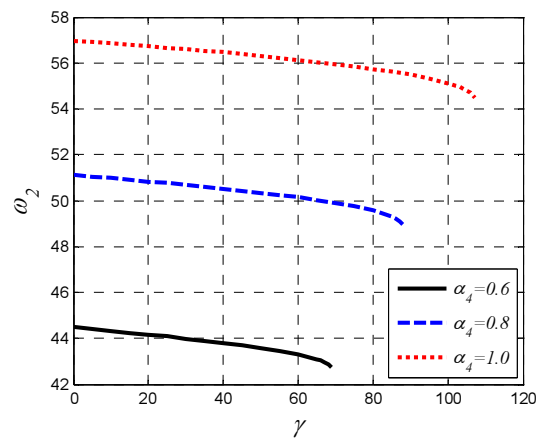

b)

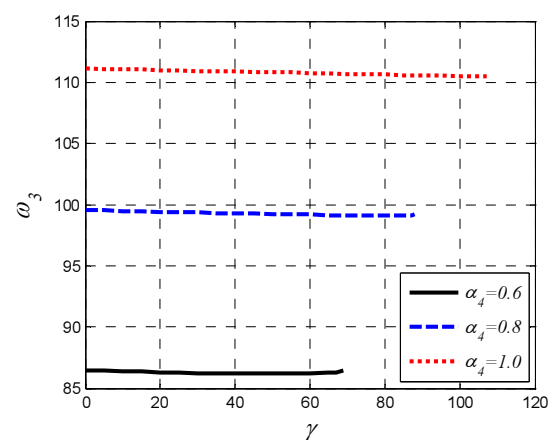

Fig. 14. Variations of (a) the second and (b) the third natural frequencies vs the Casimir force for SSA approximation and $\sigma_{2}=2$ 
Finally, to examine the effect of Casimir attraction force on the higher frequencies of nanowires, variations of the second and third natural frequencies for LSA and SSA models are shown in Figs. 11 to 14 , for some values of surface stress parameter $\sigma_{2}$ and size-effect parameter $\alpha_{4}$. As can be seen, the second natural frequency is more sensitive to the variation of Casimir parameter in comparison with the third one. In addition, one can infer that the slope of the second natural frequency increases with increasing the Casimir force, whereas the slope of the third one decreases as the Casimir force increases.

According to the illustrated results in Figs. 13 and 14, it is concluded that, when the surface stress $\sigma_{2}$ keeps constant, the second and third natural frequencies are very sensitive to variation of the scale parameter $\alpha_{4}$. Furthermore, Figs. $13 \mathrm{~b}$ and $14 \mathrm{~b}$ exhibit that the third natural frequency does not show a noticeable change when the surface stress is constant.

\section{Conclusions}

In this work we have studied the instability characteristics of Casimir-induced nanowires in the presence of microstructure size-dependency and surface effects. A non-linear governing equation of nanowires for both small and large separation gaps has been developed and the static response as well as eigenvalue problems have been presented to examine the mechanical behaviour of a considered nano-beam. It is concluded that:

1. Increasing the geometry ratio $k$ increases the instability threshold of Casimir-induced nanowires for both SSA and LSA models.

2. Increasing the surface stress parameter from negative to positive values, raises the critical Casimir value and the fundamental frequency of nanowires.

3. The fundamental frequency of nanowires increases with increasing the size-dependency parameter and the nanowire collapses onto the fixed substrate at higher values of the Casimir parameter.

4. It has been shown that the second natural frequency is more sensitive to variations of the Casimir parameter in comparison with the third one.

\section{Acknowledgment}

Dr. Hassen M. OUAKAD is grateful for the support of his funding body supported by the Deanship of Scientific Research (DSR) at King Fahd University of Petroleum and Minerals (KFUPM) (grant number SB151004).

\section{References}

[1] Cuesta, E., Mantaras, D.A., Luque, P., Alvarez, B.J., Muina, D. (2015). Dynamic Deformations in Coordinate Measuring Arms Using Virtual Simulation. International Journal of Simulation Modelling, 14(4), 609-620.

[2] Herzog, V., Zavec Pavlinic, N., Kuzmanovic, D., Buchmeister, B. (2016). Thermal Manikin and Its Stability for Accurate and Repeatable Measurements. International Journal of Simulation Modelling, 15(4), 676-687.

[3] Craighead, H.G. (2000). Nanoelectromechanical systems. Science, 290, 1532-1535.

[4] Wang, M.C.P., Gates, B.D. (2009). Directed assembly of nanowires. Materials Today, 12, 34-43.

[5] Khajeansari, A., Baradaran, G.H., Yvonnet, J. (2012). An explicit solution for bending of nanowires lying on Winkler-Pasternak elastic substrate medium based on the Euler-Bernoulli beam theory. International Journal of Engineering Science, 52, 115-128.

[6] Ternik, P., Rudolf, R. (2016). Numerical Analysis of Continuous Casting of NiTi Shape Memory Alloy. International Journal of Simulation Modelling, 15(3), 522-531. 
[7] Komeili, M., Menon, C. (2016). Robust Design of Thermally Actuated Micro-Cantilever Using Numerical Simulations. International Journal of Simulation Modelling, 15(3), 409-422.

[8] Feng, X.L., He, R., Yang, P., Roukes, M.L. (2007). Very High Frequency Silicon Nanowire Electromechanical Resonators. Nano Letters, 7(7), 1953-1959.

[9] Sedighi, H.M., Daneshmand, F., Zare, J. (2014). The influence of dispersion forces on the dynamic pull-in behavior of vibrating nano-cantilever based NEMS including fringing field effect. Archives of Civil and Mechanical Engineering, 14(4), 766-775.

[10] Farrokhabadi, A., Abadian, N., Rach, R., Abadyan, M. (2014). Theoretical modelling of the Casimir forceinduced instability in freestanding nanowires with circular cross-section. Physica E, 63, 67-80.

[11] Buks, E., Roukes, M.L. (2002). Quantum physics: Casimir force changes sign. Nature, 419, 119-120.

[12] Buks, E., Roukes, M.L. (2001). Metastability and the Casimir effect in micromechanical systems. Europhysics Letters, 54(2), 220.

[13] Zou, J., Marcet, Z., Rodriguez, A.W., Reid, M.T.H., McCauley, A.P., Kravchenko, I.I., Lu, T., Bao, Y., Johnson, S.G., Chan, H.B., (2013). Casimir forces on a silicon micromechanical chip. Nature Communications, $4,1845$.

[14] Buks, E., Roukes, M.L. (2001). Stiction, adhesion energy, and the Casimir effect in micromechanical systems. Phys. Rev. B, 63, 033402.

[15] Lombardo, F.C., Mazzitelli, F.D., Villar, P.I., (2008). Numerical evaluation of the Casimir interaction between cylinders. Physical Review D, 78, 085009.

[16] Emig, T., Jaffe, R.L., Kardar, M., Scardicchio, A. (2006). Casimir interaction between a plate and a cylinder. Physical Review Letters. 96, 080403.

[17] Bordag, M., Mohideen, U., Mostepanenko, V.M. (2001). New developments in the Casimir effect. Physics Reports, 353, 1-205.

[18] Casimir, H.B.G. (1948). On the attraction between two perfectly conducting plates. Proc. of the Koninklijke Nederlandse Akademie van Wetenschappen, 51, 793

[19] Guo, J.G., Zhao, Y.P. (2004). Influence of van der Waals and Casimir Forces on Electrostatic Torsional Actuators. Journal of Microelectromechanical Systems, 13(6), 1027.

[20] Lin, W.H., Zhao, Y.P., (2005). Nonlinear behavior for nanoscales electrostatic actuators with Casimir force. Chaos, Solitons \& Fractals, 23, 1777.

[21] Casimir, H.B.G., Polder, D. (1948). The influence of retardation of the London-van der Waals forces. Physical Review Letters, 73, 360.

[22] Teo, L.P. (2011). First analytic correction to the proximity force approximation in the Casimir effect between two parallel cylinders. Physical Review D, 84, 065027

[23] Teo, L.P. (2011). Casimir, interaction between a cylinder and a plate at finite temperature: Exact results and comparison to proximity force approximation. Physical Review D, 84, 025022.

[24] Barretta, R., Čanađija, M., Marotti de Sciarra, F. (2016). A higher-order Eringen model for BernoulliEuler nanobeams. Archive of Applied Mechanics, 86(3), 483-495.

[25] Barretta, R., Feo, L., Luciano, R., Marotti de Sciarra, F. (2015). Variational formulations for functionally graded nonlocal Bernoulli-Euler nanobeams. Composite Structures, 129, 80-89.

[26] Romano, G., Barretta, R., Diaco, M., Marotti de Sciarra, F. (2017). Constitutive boundary conditions and paradoxes in nonlocal elastic nano-beams. Int. J. Mech. Sciences., 121, 151-156.

[27] Romano, G., Barretta, R. (2017). Stress-driven versus strain-driven nonlocal integral model for elastic nanobeams, Composites Part B, DOI: 10.1016/j.compositesb.2017.01.008

[28] Čanađija, M., Barretta, R., Marotti de Sciarra, F. (2016). A gradient elasticity model of Bernoulli-Euler nanobeams in nonisothermal environments. European Journal of Mechanics - A/Solids, 55, 243-255.

[29] Sari, M., Shaat, M., Abdelkefi, A. (2017). Frequency and mode veering phenomena of axially functionally graded non-uniform beams with nonlocal residuals. Composite Structures, 163, 280-292. 
[30] Civalek, O., Demir, C, Akgöz, B. (2009). Static analysis of single walled carbon nanotubes (SWCNT) based on Eringen's nonlocal elasticity theory. Inter. J. Eng. App. Sci., 2, 47-56.

[31] Civalek, O., Demir, C. (2016). A simple mathematical model of microtubules surrounded by an elastic matrix by nonlocal finite element method. Applied Mathematics and Computation, 289, 335-352.

[32] Sedighi, H.M. (2014). Size-dependent dynamic pull-in instability of vibrating electrically actuated microbeams based on the strain gradient elasticity theory. Acta Astronautica, 95, 111-123.

[33] Shaat, M., Abdelkefi, M. (2016). On a second-order rotation gradient theory for linear elastic continua. International Journal of Engineering Science, 100, 74-98.

[34] Keivani, M., Koochi, A., Kanani, A., Mardaneh, M.R., Sedighi, H.M., Abadyan, M. (2016). Using strain gradient elasticity in conjunction with Gurtin-Murdoch theory for modeling the coupled effects of surface and size phenomena on the instability of narrow nano-switch. Proc.of the Institution of Mechanical Engineers, Part C: Journal of Mechanical Engineering Science, DOI:10.1177/0954406216642475.

[35] Akgoz, B., Civalek, O. (2013). Buckling analysis of linearly tapered micro-columns based on strain gradient elasticity. Structural Engineering and Mechanics, 48(2), 195-205.

[36] Ansari, R., Gholami, R., Sahmani, S. (2013). Size-dependent vibration of functionally graded curved microbeams based on the modified strain gradient elasticity theory. Archive of Applied Mechanics, 83(10), 1439-1449

[37] Akgoz, B, Civalek, O. (2015). A novel microstructure-dependent shear deformable beam model. International Journal of Mechanical Sciences, 99, 10-20.

[38] Daneshmand, F. (2014). Combined strain-inertia gradient elasticity in free vibration shell analysis of single walled carbon nanotubes using shell theory. Applied Mathematics and Computation, 243, 856-869.

[39] Li, L., Hu, Y., Ling, L. (2016). Wave propagation in viscoelastic single-walled carbon nanotubes with surface effect under magnetic field based on nonlocal strain gradient theory. Physica E: Low-dimensional Systems and Nanostructures, 75, 118-124.

[40] Wang, Z.Q., Zhao, Y.P., Huang, Z.P. (2010). The effects of surface tension on the elastic properties of nano structures. International Journal of Engineering Science, 48, 140-150.

[41] Dingrevillea, R., Qua, J., Cherkaoui, M. (2005). Surface free energy and its effect on the elastic behavior of nano-sized particles, wires and films. Journal of the Mechanics and Physics of Solids, 53(8), 1827-1854.

[42] Gurtin, M.E., Murdoch, A.I. (1978). Surface stress in solids. International Journal of Solids and Structures. $14,431-440$

[43] Sedighi, H.M. (2014). The influence of small scale on the pull-in behavior of nonlocal nano-bridges considering surface effect, Casimir and van der Waals attractions. International Journal of Applied Mechanics, 6(3), 22.

[44] Eltaher, M.A., Mahmoud, F.F., Assie, A.E., Meletis, E.I. (2013). Coupling effects of nonlocal and surface energy on vibration analysis of nanobeams. Applied Mathematics and Computation, 224, 760-774.

[45] Fu, Y., Zhang, J. (2011). Size-dependent pull-in phenomena in electrically actuated nanobeams incorporating surface energies. Applied Mathematical Modelling, 35(2), 941-951.

[46] Koochi, A., Hosseini-Toudeshky, H., Ovesy, H.R., Abadyan, M. (2013). Modeling the influence of surface effect on instability of nano-cantilever in presence of Van der Waals force. International Journal of Structural Stability and Dynamics, 13, 1250072.

[47] Sedighi, H.M., Bozorgmehri, A. (2016). Dynamic instability analysis of doubly clamped cylindrical nanowires in the presence of Casimir attraction and surface effects using modified couple stress theory. Acta Mechanica, 227(6), 1575-1591.

[48] Sedighi, H.M., Bozorgmehri, A. (2017). Nonlinear vibration and adhesion instability of Casimir-induced nonlocal nanowires with the consideration of surface energy. Journal of the Brazilian Society of Mechanical Sciences and Engineering, 39(2), 427-442.

[49] Beer, FP., Johnston Jr., E.R., DeWolf, J.T. (1981). Mechanics of Materials. New York: McGraw-Hill.

[50] Lu, P., He, L.H., Lee, H.P., Lu, C. (2006). Thin plate theory including surface effects. International Journal of Solids and Structures, 44, 4631-4647. 
[51] Yekrangi, A., Soroush, R. (2016). Modeling of the intermolecular Force-Induced Adhesion in Freestanding Nanostructures Made of Nano-beams. Journal of Applied and Computational Mechanics, 2(1), 1-7.

[52] Lamoreaux, S.K. (2005). The Casimir force: background, experiments, and applications. Reports on Progress in Physics, 68, 201-236.

[53] Chan, H.B., Bao, Y., Zou, J., Cirelli, R.A., Klemens, F., Mansfield, W.M., Pai, C.S. (2008). Measurements of the Casimir Force between a Gold Sphere and a Silicon Surface with Nanoscale v Trench Arrays. Physical Review Letters, 101, 030401.

[54] Li, H., Kardar, M. Fluctuation-induced forces between rough surfaces. Physical Review Letters, 67, 3275.

[55] Buscher, R., Emig, T. (2005). Geometry and Spectrum of Casimir Forces. Physical Review Letters, 94, 133901.

[56] Rahi, S.J., Emig, T., Jaffe, R.L., Kardar, M. (2008). Casimir forces between cylinders and plates. Physical Review A, 78, 012104.

[57] Bulgac, A., Magierski, P., Wirzba, A. (2006). Scalar Casimir effect between Dirichlet spheres or a plate and a sphere. Physical Review D, 73, 025007.

[58] Lam, D.C.C., Yang, F., Chong, A.C.M., et al. (2003). Experiments and theory in strain gradient elasticity. Journal of the Mechanics and Physics of Solids, 51(1), 477-508.

[59] Shaat, M., Mohamed, S.A. (2014). Nonlinear-electrostatic analysis of micro-actuated beams based on couple stress and surface elasticity theories. International Journal of Mechanical Sciences, 84, 208-217.

[60] Batra, R.C., Porfiri, M., Spinello, D. (2008). Vibrations of narrow microbeams predeformed by an electric field. Journal of Sound and Vibration, 309, 600-612.

[61] Soltani, P., Kassaei, A., Taherian, M.M. (2014). Nonlinear and quasi-linear behavior of a curved carbon nanotube vibrating in an electric force field; an analytical approach. Acta Mechanica Solida Sinica, 27(1), $97-110$.

[62] Rahmani, O., Pedram, O. (2014). Analysis and modeling the size effect on vibration of functionally graded nanobeams based on nonlocal Timoshenko beam theory. International Journal of Engineering Science, 77, $55-70$.

[63] Younis, M.I., Abdel-Rahman, E.M., Nayfeh, A.H. (2003). A Reduced-order model for electrically actuated microbeam-based MEMS. J. Microelectromech. Syst., 12, 672-680.

[64] Whittaker, E.T., Robinson, G. (1967). The Newton-Raphson Method, $\S 44$ in the Calculus of Observations: A Treatise on Numerical Mathematics, (4th ed.), NY: Dover, 84-87.

[65] Nayfeh, A.H., Balachandran, B. (1995). Applied Nonlinear Dynamics. Wiley, New York.

[66] Farrokhabadi, A., et al. (2014). Theoretical modeling of the Casimir force-induced instability in freestanding nanowires with circular cross-section. Physica E: Low-dimensional Systems and Nanostructures, 63, 67-80. 